\title{
Calidad informativa en la era de la digitalización: Fundamentos profesionales vs. infopolución
} Informative Quality in the era of digitalization: Professional foundations vs. Infopollution

Fechas | En edición: 27/05/2019 - Publicación final: 01/07/2019

\section{Dra. Sara OSUNA-ACEDO}

Universidad Nacional de Educación a Distancia. España. sosuna@edu.uned.es

\section{Calidad informativa en la era de la digitalización: Fundamentos profesionales vs. infopolución}

Carmen MARTA-LAZO (Editora).

Madrid: Dykinson, 2018.

208 páginas

ISBN: 978-84-9148-670-1

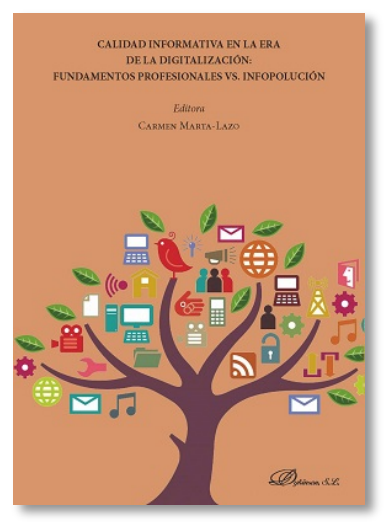

\section{Resumen}

El libro Calidad informativa en la era de la digitalización: Fundamentos profesionales vs. Infopolución presenta el trabajo pormenorizado de reconocidos investigadores que presentan sus avances en el área de la comunicación. El libro, estructurado en un prólogo y 12 capítulos, está dirigido a los docente, investigadores y profesionales del periodismo y de la comunicación digital. Los autores se centran en la calidad de la información en la era actual, caracterizada por la infopolución, la postverdad y la manipulación informativa. En todos los capítulos se tratan cada uno de los temas que preocupan acerca del proceso de comunicación en los tiempos actuales, sin perder la perspectiva del humanismo digital. Por ello, este libro está escrito teniendo como eje central a una ciudadanía digital, participativa, intercreativa y protagonista de sus actos comunicativos en los entornos que proporcionan las Tecnologías de la Relación, la Información y la Comunicación (TRIC).

\section{Palabras clave}

Calidad informativa; Educomunicación y competencias profesionales de los informadores; Infopolución; Manipulación informativa; Postverdad; TRIC.

\begin{abstract}
Informative Quality in the era of digitalization: Professional foundations vs. Infopollution presents the detailed work of renown researchers showing their progress in the communication area. The book, structured in a prologue and 12 chapters, is aimed at teachers, researchers and professionals in journalism and digital communication. The authors focus on the quality of information in the current era, characterized by information pollution, post-truth and informative manipulation. Each chapter deals with a different issue concerning communication process in the current times, without losing the perspective of digital humanism. Therefore, this book is written focusing on a digital, participatory, intercreative citizenship and protagonist of its communicative acts in the environments provided by the Relationship, Information and Communication Technologies (RICT).
\end{abstract}

\section{Keywords}

Informative quality; Educommunication and professional skills of the informants; Infopolution; Informative manipulation; Post-truth; RICT. 
Calidad informativa en la era de la digitalización: Fundamentos profesionales vs. Infopolución, editado por la prestigiosa educomunicadora Carmen Marta-Lazo, es una importante publicación que da explicación a muchos interrogantes sobre el mundo del periodismo y la comunicación digital. Los cambios que se han producido en la sociedad digital han roto y transformado los modos de consumo de información, apareciendo nuevos términos peyorativos como infopolución, manipulación informativa, postverdad o fake news, entre otros, que necesitan una respuesta positiva desde un ejercicio periodístico creíble, crítico y comprometido.

Los distintos capítulos que componen el libro giran en torno a dos grandes núcleos temáticos: la calidad informativa en los medios sociales de comunicación y en plataformas móviles, por un lado, y la relación de la Comunicación con otras áreas sociales como la Economía y la Política, por otro.

Respecto al primer núcleo de la calidad informativa, en el capítulo 1 se realiza un diagnóstico sobre la credibilidad informativa en los nuevos medios y la pérdida de confianza a nivel general. Después de analizar los motivos que ocasionan la falta de credibilidad que siente la ciudadanía española y europea en la información que recibe, se concluye con el impacto negativo que ocasiona en el desarrollo social y democrático. Urge actuar para eliminar el descrédito, la fragmentación de la información y la imposibilidad de verificar sus fuentes, con lo que este capítulo propone actuaciones concretas que garanticen la calidad informativa. Precisamente, el capítulo 3 se centra en la hiperfragmentación del discurso que ofrecen las metapantallas, centrándose en el caso de Youtube.

En el capítulo 10 se analizan pormenorizadamente los problemas coyunturales que acontecen en la profesión periodística dentro de la era digital. La infopolución, la manipulación informativa, las fake news, los bots se convierten en el centro de estudio para obtener lo positivo de estos hechos, que nos llevarán hacia un periodismo confiable. Asimismo, en el capítulo 11 se profundiza en la manera en la que se están abriendo nuevas brechas informativas, que requieren las mediaciones oportunas para la inclusión digital de toda la ciudadanía. De aquí se desprende que se generan nuevas diferencias peyorativas entre los países prósperos y los que están aún en desarrollo. La diferencia fundamental no es tanto la posesión de tecnologías, sino la alfabetización mediática de la población que recibe la información.

Por ello, en el capítulo 7 se fundamenta el contexto TRIC, que implica una comprensión de las tecnologías digitales basadas en el Factor Relacional. Esto quiere decir que las tecnologías como soporte ceden el protagonismo a la ciudadanía que participa en ellas. Por esto, la cultura de nuestra era recibe el nombre de Cultura de la Participación. Pero, ¿̇cómo puede participar la ciudadanía de forma implicada, responsable y crítica sobre la información que recibe? La respuesta del capítulo es mediante la alfabetización mediática y las competencias mediáticas y digitales. Siguiendo los preceptos educomunicativos, en el capítulo 8 se analizan las posibilidades de las apps para conseguir un aprendizaje dialógico, que debe responder a la adquisición de las competencias mediáticas exigidas por la sociedad digital. Por su parte, el capítulo 9 profundiza en las competencias mediáticas concretas que necesitan los profesionales del periodismo y la comunicación digital, así como la responsabilidad de la universidad que titula a dichos profesionales. La Sociedad Digital está encomendando a estos futuros profesionales que realicen las mediaciones oportunas para que la ciudadanía valore una información veraz en tiempos de infopolución y de redes sociales. En este sentido, se propone la salvaguardia de la esencia de la profesión periodística, independientemente de las tecnologías utilizadas y las posibilidades narrativas que proporcione.

El capítulo 12 es el que pone el broche al primer núcleo temático del libro sobre la calidad informativa, abordando el entretenimiento de calidad en una sociedad donde se ensalza el ocio audiovisual y digital. De nuevo, se propone que la Educación Mediática tome las riendas ante el universo narrativo y gamificado que se tiene al alcance. Es necesario velar por la calidad del proceso formativo de periodistas y comunicadores con estas nuevas realidades, donde la tecnología puede deslumbrar y dar una idea de no accesibilidad o alejamiento de la ciudadanía.

Los capítulos 2, 4, 5 y 6 abordan el segundo gran núcleo temático del libro que nos aporta los resultados de las investigaciones más recientes acerca de la relación de la Comunicación con otras áreas. Por su parte, el capítulo 2 analiza el proceso de adaptación de la Comunicación a los nuevos modelos de negocio y reconversión de las empresas de comunicación en la actualidad. Nos presenta la profunda transformación de los medios producidos por la convergencia tecnológica. En el capítulo 4, se evalúan las implicaciones que tiene el registro, almacenamiento, procesamiento y generación de nuevos datos analizados en grandes cantidades. La datificación, el big data y la inteligencia artificial tienen unas ventajas y desventajas que analiza este cuarto artículo.

El capítulo 5 estudia el caso concreto de la Comunicación Política y la Opinión Pública, analizándose campañas políticas concretas para presentar sus estrategias comunicativas. Sus autores llegan a la conclusión de que la ciberpolítica bien entendida puede llegar a construir una ciber-opinión que potencie 
intercambios de información veraz y de calidad que, a su vez, nos lleve a la construcción de una ciberdemocracia. Por último, el capítulo 6 se adentra en el análisis de la gestión de la comunicación de las instituciones. La dotación de tecnologías ocasiona un gran reto para avanzar en la comunicación organizacional que promueva la cooperación en los aspectos comunicativos.

En definitiva, este libro tan innovador nos abre una nueva perspectiva del periodismo y nos deja con ganas de leer más sobre esta visión crítica y actual con nuevos roles de la ciudadanía en el proceso informativo y la vuelta a los fundamentos del tratamiento de la información en una era de tan profundos cambios. 